\title{
ENTORNOS PERSONALES DE APRENDIZAJE EN EL ESPACIO EUROPEO DE EDUCACIÓN SUPERIOR
}

\author{
(PERSONAL LEARNING ENVIRONMENTS IN THE EUROPEAN HIGHER EDUCATION)
}

\author{
Inmaculada Tello Díaz-Maroto \\ Lourdes de Miguel Barcala \\ María Dolores López Carrillo \\ Escuela Universitaria Cardenal Cisneros (España)
}

\section{RESUMEN}

En la actualidad existen multitud de plataformas de formación, muchas de las cuales no siempre responden a las necesidades de una institución de formación a distancia. El objetivo de esta ponencia es analizar aquellos aspectos importantes a evaluar a la hora de seleccionar una buena plataforma de formación on-line, teniendo en cuenta las nuevas características de la educación superior basadas en las ideas del Plan Bolonia. Para ello, se hace hincapié en la importancia de Internet para la formación a distancia, se enfatiza la enseñanza y evaluación por competencias y se analizan los aspectos a tener en cuenta en una plataforma. Si deseamos tener enseñanza de calidad a través de plataformas de teleaprendizaje debemos evaluar la funcionalidad, los aspectos técnicos y estéticos y los aspectos pedagógicos de las plataformas utilizadas.

Palabras clave: plataformas, e-learning, formación a distancia, calidad, competencias, evaluación.

\begin{abstract}
Today, there are many training platforms, some of which that do not always have what distance learning institutions need. The aim of this paper is to analyze the important aspects which need to be considered when selecting a good platform for online training, based on the latest trends in higher education. For this purpose, the article discusses the importance of the Internet for distance learning, teacher observation, and competency assessment. It also discusses the things to consider in evaluating the platforms. If we want to have quality education through distance learning platforms, we must evaluate the functionality of the platform: its technical, esthetic and educational aspects.
\end{abstract}

Keywords: platform, e-learning, distance learning, quality, competences, evaluation. 
En la actual sociedad de la información y la comunicación se hacen necesarios varios cambios que hagan posible la adaptación a las nuevas características de la sociedad. Por ello, nos encontramos en una sociedad en continuo cambio que cada vez requiere de nosotros mayores actualizaciones. En este caso, nos vamos a centrar en dos aspectos de cambio muy importantes en la sociedad actual, por un lado la nueva concepción de la educación superior basada en la concepción de la Unión Europea, y por otro lado la necesaria incorporación de la educación al mundo digital.

Pero estos dos cambios tan necesarios en la educación superior son tan complementarios que debemos intentar que uno facilite al otro y viceversa, ya que tienen muchas características coincidentes. Por ejemplo, si queremos que nuestros alumnos trabajen en pequeños grupos o seminarios de forma práctica, podemos facilitarles muchas herramientas digitales que harán más práctico y cercano dicho trabajo por seminarios.

Con esa idea parte este artículo, con el ánimo de extraer los beneficios de ambos cambios metodológicos de la educación superior (competencias y TIC) y hacer un único cambio, facilitando al profesorado la concepción y trabajo de ambas vertientes.

Para la presentación de este trabajo comenzaremos analizando la importancia que Internet está teniendo en la educación en los últimos años, sea cual sea la modalidad de enseñanza y aprendizaje utilizada.

A continuación destacaremos la necesidad de evaluar la calidad de la educación ya sea en formación presencial o formación virtual, así como la necesidad de evaluar los recursos utilizados en dicha educación.

Finalmente, nos centraremos en resaltar los aspectos necesarios para tener un espacio personal de aprendizaje de calidad en una plataforma virtual en función de las características de la educación superior según el Plan Bolonia.

\section{INTERNET EN LA EDUCACIÓN}

Hemos podido observar la aplicación que las TIC tienen en el aprendizaje a lo largo de toda la vida (formación de profesionales en activo), siendo para ello la educación a distancia, la metodología de enseñanza y aprendizaje más adecuada por su flexibilidad de horarios y espacios físicos, entre otras cosas. De este modo, el profesor de hoy en día se encuentra con una metodología de enseñanza a distancia que cada vez ha ido teniendo más demanda. Esta, en sus orígenes, se basaba en 
una educación a través de materiales que se remitían al estudiante por correo ordinario, y a través de una comunicación por teléfono con el tutor y profesor del curso o asignatura. Este tipo de educación ha evolucionado desde el surgimiento y divulgación de los productos multimedia primero, y de Internet poco después, como apoyo a la enseñanza y al aprendizaje.

Pero el uso educativo de este recurso no solo tiene cabida en la educación a distancia, donde es innegable por sus potencialidades, sino también en otros modelos de enseñanza. Podemos distinguir al menos tres grandes usos de Internet en los ámbitos de la educación y la formación:

- Internet como apoyo a la enseñanza presencial: En este caso la educación es presencial, pero se utilizan algunas potencialidades que nos ofrece esta herramienta, como por ejemplo para buscar información relevante y de actualidad, para intercambiar opiniones sobre algún tema educativo en foros o chat destinados a este objetivo, etc.

- Enseñanza semipresencial a través de Internet: En este tipo de enseñanza, se hace un mayor uso de las potencialidades que Internet tiene. En la enseñanza semipresencial se transmiten los contenidos a través de la red, además de contar, como en el caso anterior, de herramientas de comunicación, y de otras muchas herramientas que se ponen a disposición de los alumnos para que su estudio a distancia tenga una mayor similitud al aula tradicional (se disponen de herramientas como agenda, tablón de anuncios, fichas personales de alumnos y profesores...). Además, se programan encuentros presenciales que posibilitan las relaciones interpersonales directas entre los participantes del curso o asignatura. Dentro de esta modalidad de enseñanza nos encontramos con la metodología mixta o blended Learning y el e-learning. Ambas metodologías se diferencian en el grado de presencialidad, pues mientras en la primera nos encontramos con una distribución de alrededor del 50\% presencial y alrededor del 50\% on-line, en el e-learning nos encontramos con menos porcentaje de presencialidad, siendo el mínimo considerable 2 o 3 sesiones presenciales, pues si no hay presencialidad pasaría a ser formación virtual u on-line. A su vez, en función del grado de presencialidad variará el enfoque y actividades de las sesiones presenciales, pudiendo destinarse a estimular las relaciones entre participantes, si es pequeño el número de dichas sesiones, o a la impartición de algunos conocimientos, solución de dudas, corrección de actividades..., si contamos con un mayor número de sesiones presenciales. 
- Enseñanza virtual con Internet: En el caso de la enseñanza virtual se utilizan la mayoría, si no son todas, de las potencialidades educativas que proporciona Internet. En este caso, se utilizaría este medio tecnológico como un sistema educativo integrado impartiéndose toda la enseñanza a distancia, sin ningún encuentro presencial.

Como vemos, Internet tiene múltiples posibilidades de actuación en la educación. Ya mencionaba Gisbert y otros (1998)1: "si Internet es un medio de comunicación y la comunicación entre las personas es la base de todo proceso educativo...”, podemos entender el gran impacto que esta está teniendo en la educación.

Las potencialidades que Internet ofrece al ámbito educativo son inmensas, la clave está en cómo utilizar dicho recurso adecuadamente, cómo aprovechar las potencialidades de las que hablábamos para mejorar el proceso de enseñanza y aprendizaje.

Algunas de dichas potencialidades vienen enumeradas por Roig (2002) y nos hemos permitido agruparlas en el siguiente cuadro:

\begin{tabular}{|l|}
\hline \multicolumn{1}{|c|}{ En general } \\
\hline - Su gran inmensidad constituye por sí sola una potencialidad de este recurso. \\
\hline - Sus contenidos se actualizan continuamente y es posible acceder a ellos con rapidez. \\
\hline - Facilita el conocimiento de otras realidades y culturas. \\
\hline - El tiempo y el espacio ya no tienen la relevancia de la escuela tradicional. \\
\hline $\begin{array}{l}\text { - Contribuye a evitar el aislamiento al tiempo que favorece el trabajo colaborativo y la } \\
\text { educación flexible. }\end{array}$ \\
\hline $\begin{array}{l}\text { - Permite la consulta a expertos o profesionales a fin de resolver problemas o profundización } \\
\text { en contenidos de investigación. }\end{array}$ \\
\hline $\begin{array}{l}\text { - Aporta la posibilidad de recopilar ingente información relacionada con el tema, contenido } \\
\text { o habilidad que se trate en cada momento. }\end{array}$ \\
\hline - Permite encontrar información de primera mano. \\
\hline - Facilita la conexión con los autores de obras de áreas diversas y obtener información \\
directa de ellas. \\
\hline - Permite encontrar fundamentos y complementos de las ideas propias. \\
\hline - Permite colaborar con otros docentes en la elaboración de proyectos y actividades. \\
\hline
\end{tabular}




\begin{tabular}{|l|}
\hline $\begin{array}{l}\text { - Permite encontrar y compartir planificaciones curriculares que apoyen el desarrollo de } \\
\text { una clase. }\end{array}$ \\
\hline $\begin{array}{l}\text { - Facilita descubrir oportunidades de desarrollo profesional accediendo a material e } \\
\text { información actualizados. }\end{array}$ \\
\hline - Permite contactar con el resto de la comunidad (padres, profesores, etc.). \\
\hline \multicolumn{1}{c|}{ Para los discentes } \\
\hline $\begin{array}{l}\text { - Permite aprender sobre una materia, tema o habilidad, y de un modo contrastado, con } \\
\text { diversas perspectivas. }\end{array}$ \\
\hline - Permite investigar temas que les sean de interés o según las indicaciones de los docentes. \\
\hline - Permite desarrollar estrategias de investigación. \\
\hline - Entender acontecimientos, hechos, datos, etc. accediendo a información de primera mano. \\
\hline - Crear proyectos colaborativos desarrollados con las herramientas o aplicaciones de \\
Internet. \\
\hline - Participar en proyectos colaborativos desarrollados a través de la red y originados en otros \\
centros. \\
\hline - Conectarse con estudiantes de otros lugares, lenguas, culturas, etnias, etc., es decir, \\
conectarse con la diversidad y poder no solo concienciarse de ello, sino desarrollar \\
tolerancia hacia la diferencia, respeto y, mejor, articulación. \\
\hline - Contactar con autores de obras de diversas áreas. \\
\hline
\end{tabular}

Tabla 1. Potencialidades de Internet en la educación

Dicho autor, además, hace referencia a la importancia que en educación se está dando a la utilización de Internet, como con anterioridad quedaba también reflejado en el trabajo desarrollado por la Comisión de las Comunidades Europeas (2001) ${ }^{2}$, concretamente en la iniciativa eLearning (complementaria del Plan de Acción Global eEurope), fijando dentro de ella los siguientes objetivos (recogidos en parte también en eEurope):

- Dotar de acceso a Internet y de recursos multimediales a todas las escuelas de aquí a finales de 2001, y a todas las salas de clase (Internet rápido) de aquí a finales de 2002.

- Conectar progresivamente a las escuelas a las redes de investigación, de aquí a finales de 2002.

- Alcanzar la relación de 5 a 15 alumnos por ordenador multimedia en 2004. 
- Garantizar la disponibilidad de servicios de apoyo y de recursos educativos en Internet, así como de plataformas de aprendizaje en línea destinadas a profesores, alumnos y padres, de aquí a finales de 2002.

- Apoyar la evolución de programas escolares para tener en cuenta los nuevos métodos de aprendizaje y utilización de las TIC, de aquí a finales de 2002.

En muchas ocasiones nos encontramos con centros de enseñanza que disponen de grandes infraestructuras y conexión a Internet, pero no saben utilizarlos pedagógicamente. ¿¿De qué sirve entonces tener los medios adecuados si no se tiene la pedagogía adecuada para utilizarlos?

\section{NECESIDAD DE EVALUAR LA CALIDAD DE LA EDUCACIÓN}

Como hemos podido observar en los apartados anteriores, nos encontramos en una sociedad del conocimiento y de la información en la que surgen nuevas necesidades que deben ser resueltas, y en las que la educación y la formación juegan un papel transversal. Así también lo menciona Blázquez $^{3}$ (1998, p. 91) cuando afirma: "la sociedad de la información demanda de la institución escolar que prepare a sus alumnos para convivir con ella".

Además, se trata de una sociedad del cambio en la que los avances tecnológicos evolucionan a una velocidad estrepitosa. Dichos avances van creando a su vez, en el ámbito educativo, nuevas formas de transmitir los conocimientos que modifican la metodología de enseñanza y aprendizaje. Con ello no queremos decir que se vaya sustituyendo una metodología por otra, sino que ambas formas de enseñar y aprender se solapan, propiciando nuevas posibilidades de formarse.

La incorporación de dichas tecnologías es un hecho ya patente en la realidad y que va implantándose en cada vez más ámbitos de la vida. Por ello no debemos negarles el acceso, sino luchar porque su incorporación en nuestra sociedad sea lo más satisfactoria posible, y aprovechar así todas sus potencialidades. Y para ello es necesario que las nuevas tecnologías de la información y la comunicación sean utilizadas de forma adecuada, mejorando la calidad de vida.

Desde el ámbito de la educación, el cual funciona como principal pilar de la formación integral de los individuos, debemos conseguir que la incorporación de dichas tecnologías, y entre ellas Internet, sea lo más satisfactoria posible. Formulábamos en el apartado anterior una duda que debemos tener en cuenta: ¿De 
qué sirve entonces tener los medios adecuados si no se tiene la pedagogía adecuada para utilizarlos?

Uno de los pasos, pues, para conseguir una adecuada incorporación de las TIC a la educación es evaluar no solo los aspectos técnicos, sino también los pedagógicos de cada medio utilizado. Entre las Tecnologías de la Información y la Comunicación nos encontramos con una inmensidad de recursos. Nosotros nos vamos a centrar en analizar aquellos aspectos que hacen que los programas de formación impartidos a través de Internet sean de calidad. Son muchos los cursos que actualmente se imparten a través de la red, y algunos no tienen en cuenta unos determinados estándares de calidad en su diseño y elaboración.

Cada vez son más los profesionales que se interesan por una formación a distancia, y más las organizaciones e instituciones de educación a distancia que se preocupan por implantar en sus programas la formación on-line.

Ello lleva consigo la lucha por mejorar, idea que alberga la importancia de la calidad de los productos ofertados. Por ello, queremos plantear la necesidad de evaluar la calidad de esa nueva forma de enseñar y aprender a distancia que tiene múltiples denominaciones: formación on-line, formación a través de Internet, a través de la red, e-learning, etc. Pero no se trata solo de evaluar el producto a través de la satisfacción de sus usuarios, sino también de llevar a una balanza los pros y los contras del mismo, posibilitando así su modificación y mejora, de poder tomar decisiones acertadas en cuanto a sus posibles modificaciones, en definitiva, de poder ofertar una formación cada vez de mayor calidad.

Si logramos que los programas formativos ofertados a través de Internet estén sujetos a unos determinados estándares de calidad así como los espacios virtuales a través de los cuales son ofertados, lograremos que dicho tipo de formación sea acogido de forma más positiva por la sociedad, y que se pierdan muchos de los miedos presentes aún en algunos profesionales de la educación con respecto al uso de las denominadas tecnologías de la información y la comunicación en el ámbito formativo.

Esta preocupación por la calidad de la educación y la formación es paralela a la preocupación por incorporar las tecnologías de la información y la comunicación en la educación. Así queda reflejado también en la Recomendación del Parlamento Europeo y del Consejo relativa a la cooperación europea en materia de evaluación de la calidad de la educación escolar (2001/166/CE) ${ }^{4}$. 
“En la directriz no 8 se hace referencia explícita a la necesidad de desarrollar conocimientos informáticos, de equiparar las escuelas con material informático y de facilitar el acceso de los estudiantes a Internet antes de que finalice el año 2002, lo que deberá incidir positivamente en la calidad de la educación y preparar a los jóvenes para la era digital".

La necesidad de obtener ciertos niveles de calidad constituye uno de los objetivos de los sistemas de educación y formación señalados por la Comisión de las Comunidades Europeas ${ }^{5}$.

\begin{tabular}{|c|c|}
\hline Objetivo 1: & $\begin{array}{l}\text { MEJORAR LA CALIDAD Y LA EFICACIA DE LOS SISTEMAS DE } \\
\text { EDUCACIÓN Y FORMACIÓN EN LA UNIÓN EUROPEA }\end{array}$ \\
\hline 1.1 & Mejorar La educación y la formación de profesores y formadores. \\
\hline 1.2 & $\begin{array}{l}\text { Desarrollar las aptitudes necesarias para la sociedad del conocimiento: } \\
\text { mejorar la capacidad de lectura, escritura y cálculo; actualizar la definición } \\
\text { de las competencias necesarias en la sociedad del conocimiento; y mantener } \\
\text { la capacidad de aprender. }\end{array}$ \\
\hline $\mathbf{1 . 3}$ & $\begin{array}{l}\text { Garantizar el acceso de todos a las TIC: equipar a las escuelas y centros de } \\
\text { formación; involucrar a los profesores y los formadores; utilizar las redes y } \\
\text { los recursos disponibles. }\end{array}$ \\
\hline 1.4 & Atraer a más jóvenes a los estudios científicos y técnicos. \\
\hline 1.5 & $\begin{array}{l}\text { Aprovechar al máximo los recursos. Mejorar la garantía de calidad; garantizar } \\
\text { la utilización eficaz de los recursos. }\end{array}$ \\
\hline Objetivo 2: & $\begin{array}{l}\text { FACILITAR EL ACCESO DE TODOS A LOS SISTEMAS DE } \\
\text { EDUCACIÓN Y FORMACIÓN }\end{array}$ \\
\hline 2.1 & Entorno Abierto para el aprendizaje. \\
\hline 2.2 & Hacer más atractivo el aprendizaje. \\
\hline $\mathbf{2 . 3}$ & Apoyar la ciudadanía activa: igualdad de oportunidades y cohesión social. \\
\hline Objetivo 3: & $\begin{array}{l}\text { ABRIR A UN MUNDO MÁS AMPLIO LOS SISTEMAS DE } \\
\text { EDUCACIÓN Y FORMACIÓN }\end{array}$ \\
\hline 3.1 & $\begin{array}{l}\text { Reforzar Los vínculos con el mundo del trabajo y con el mundo de la } \\
\text { investigación y la sociedad en general. }\end{array}$ \\
\hline 3.2 & Desarrollar el espíritu de empresa. \\
\hline $\mathbf{3 \cdot 3}$ & Mejorar el aprendizaje de idiomas extranjeros. \\
\hline 3.4 & Aumentar la movilidad y los intercambios. \\
\hline 3.5 & Reforzar la cooperación europea. \\
\hline
\end{tabular}

Tabla 2. Objetivos de los sistemas de educación y formación 
Si bien es cierto que todos los objetivos mencionados por la Comisión de las Comunidades Europeas son de máxima importancia, podemos resaltar el objetivo 1.5 "Aprovechar al máximo los recursos" por su relación directa con el tema. Dicho objetivo se concreta de forma específica en los siguientes:

- Garantizar una distribución y una utilización equitativas y eficaces de los recursos financieros en el sistema de educación y formación.

- Apoyar los sistemas de evaluación y aseguramiento de la calidad utilizando indicadores y análisis comparativos.

- Analizar las posibilidades de las asociaciones del sector público y privado.

- Realizar análisis de la relación coste-beneficio de las inversiones en educación y formación.

Como se observa, se da gran importancia desde dicha Comisión a la evaluación y aseguramiento de la calidad, puesto que es uno de los aspectos a tener en cuenta para poder proporcionar a nuestros alumnos una adecuada educación y formación. Hay que recalcar que no solo debe existir calidad en la formación presencial, sino en todo tipo de formación, aunque es en este en el que más avanzados se encuentran los estudios sobre indicadores de calidad.

\section{LAS PLATAFORMAS DIGITALES EN EL PLAN BOLONIA}

\section{Conceptualización de competencias}

En la sociedad actual, el tema candente en cualquier etapa de educación es la programación y evaluación por competencias. Esta importancia otorgada a las competencias se deriva de la Comisión Europea, que apuesta por homogeneizar la educación de diversos países y tener siempre presente la práctica laboral en la educación, sobre todo en los niveles superiores de educación.

Al hablar de competencias podemos citar a McClelland como el precursor al introducir en su obra Testing for competence rather tan intelligences (1973) dicho concepto. Plantea una crítica a la relación de la formación académica con el contexto laboral. En este sentido, realiza un estudio centrado en la detección de los rasgos de los emprendedores exitosos, para establecer aquellos factores que inciden en 
el desempeño profesional. En base a los resultados de este estudio se establece el concepto de competencia.

En esta misma línea y con el fin de determinar qué destrezas necesitan los jóvenes para incorporarse al mundo laboral, el secretario de Trabajo del Gobierno Federal de los EE.UU. encargó una comisión con la finalidad principal de estimular una economía altamente eficiente basada en puestos de trabajo altamente cualificados. Los resultados de la comisión se publicaron en 1992 con el nombre de informe SCANS (Secretary's Commision on Achieving Necessary Skills) resaltando tres tipologías de competencias fundamentales:

- Destrezas básicas: lectura, escritura, aritmética y expresión oral.

- Capacidad de razonamiento: pensamiento creativo, toma de decisiones informadas, resolución de problemas, capacidad de visualizar situaciones complejas y de aprender a aprender.

- Cualidades personales: responsabilidad individual, autoestima, sociabilidad, capacidad de organización e integridad personal.

Posteriormente, en el Informe Delors de la UNESCO (1996), "La Educación encierra un tesoro", se establece una nueva definición de educación basada en cuatro pilares fundamentales: aprender a ser, aprender a conocer, aprender a hacer, aprender a convivir.

Estos cuatro pilares son los puntos cardinales que propone la UNESCO para el desarrollo de las distintas políticas educativas, tanto en la educación básica como para la formación profesional y la educación superior. En este documento aparece el término competencias, vinculado a la aplicación de la noción de cualificación profesional, en el pilar "Aprender a hacer: de la noción de calificación a la de competencia”.

En 2001, la Comisión Europea estableció un grupo de trabajo constituido por expertos nacionales con el encargo de definir el concepto de competencia clave y proponer un común denominador que fuera asumido por los países de la Unión Europea. Como contribución a este proceso, la Red Europea de Información sobre Educación (Eurydice) publicó en el año 2002 un estudio comparativo "Las Competencias Clave: un concepto de expansión dentro de la educación general 
obligatoria”, realizado entre 15 países europeos sobre la utilización del término "competencia clave" en los currículos de la educación obligatoria.

Otro hito destacado del proceso de construcción de la noción de competencias básicas lo aporta la OCDE a través del proyecto DeSeCo (Definición y Selección de Competencias). DeSeCo categoriza las competencias clave en tres ámbitos:

- Actuar de manera autónoma.

o Capacidad para defender y afirmar sus derechos, sus intereses, sus responsabilidades, sus límites y sus necesidades.

- Capacidad de concebir y de realizar proyectos de vida y proyectos personales.

Capacidad de actuar en el conjunto de la situación.

- Utilizar herramientas de forma interactiva.

- Capacidad de uso del lenguaje, los símbolos y los textos de modo interactivo.

o Capacidad de utilizar el saber y la información de forma interactiva.

o Capacidad de uso de la tecnología de manera interactiva.

- Funcionar (intervenir) en grupos socialmente heterogéneos.

- Capacidad de mantener buenas relaciones con los demás.

- Capacidad de cooperación.

o Capacidad de gestionar y resolver conflictos.

El referente más directo en el proceso de concreción de las competencias básicas en nuestra vigente legislación educativa, es la recomendación comunitaria "Competencias clave para el aprendizaje permanente: un marco de referencia europeo". Dicho documento, fruto de la llamada Estrategia de Lisboa (2000), fue elaborado en el año 2005 a propuesta de la Comisión Europea y aprobado por el Parlamento Europeo y el Consejo de la Unión Europea en diciembre de 2006. Aunque el tema de las competencias ya se lleva trabajando como hemos mencionado 
al inicio desde hace unos años, por ejemplo Spencer y Spencer ya hablaban en 1993 de las competencias como "una característica subyacente en una persona que está causalmente relacionada con el desempeño, referido a un criterio superior o efectivo, en un trabajo o situación".

Cada una de las áreas curriculares debe contribuir al desarrollo de las diferentes competencias y, a su vez, cada una de las competencias básicas se alcanzará como consecuencia del trabajo en las distintas áreas o materias. De forma análoga, las competencias básicas están también interrelacionadas entre ellas, de manera que, salvo en casos muy concretos, la mayoría de escenarios y actividades de aprendizaje deben comportar el trabajo de diversas competencias de forma integrada y continua.

\section{Cambio metodológico según el Espacio Europeo de Educación Superior}

Es necesario, pues, un "cambio de paradigma del proceso enseñanza-aprendizaje" en el que debemos comenzar por preparar a los profesores de las distintas etapas educativas, entre ellas, la universitaria, para poder enseñary evaluar por competencias con el fin de renovar la Enseñanza Superior. Para ello, se les debe proporcionar tanto formación como los recursos que les faciliten esta labor de repensar el proceso de enseñanza y aprendizaje.

En palabras de Miguel Díaz (2005, p. 19): "el reto es diseñar unas modalidades y metodologías de trabajo del profesor y de los alumnos que sean adecuados para que un "estudiante medio" pueda conseguir las competencias que se proponen como metas del aprendizaje. Por ello, una vez establecidas las competencias a alcanzar, la planificación de una materia exige precisar las modalidades y metodologías de enseñanza-aprendizaje adecuadas para su adquisición así como los criterios y procedimientos de evaluación que vamos a utilizar para comprobar si se han adquirido realmente".

Por lo tanto, debemos entrelazar el tipo de enseñanza con la metodología y modalidad educativa con las competencias que queremos desarrollar en nuestros alumnos, y con la forma de evaluar si las han adquirido. Utilizando el símil del ordenador, Massot y Feisthammel (2003), señalan: "la lectura de las líneas de programación de un software complejo es prácticamente imposible (excepto para un experto de alto nivel) y no permite hacerse una representación del trabajo real que puede realizar dicho software. Sólo cuando enfrenta el software a una tarea real, el usuario puede hacerse una idea de su adecuación, su rapidez y su comodidad". 
En consecuencia, "una persona dispone de una competencia en una situación dada. Si la confrontación con el ejercicio real no se produce, la competencia no es perceptible o no se pone a prueba. Solo existe la competencia si se vincula a un objeto o una situación”.

Con ello se observa que no basta con formar al alumno en determinados conocimientos, habilidades y promover en él/ella determinadas actitudes o valores, sino que es necesario, además, favorecer el crecimiento continuo de esas características y situar a los discentes ante diversas situaciones de estudio y trabajo similares a las que puede encontrar en la práctica de su profesión.

Para lograr el desarrollo de las competencias necesarias en los alumnos universitarios, se plantea desde Bolonia utilizar una diversidad de metodologías y modalidades de enseñanza: clases teóricas, seminarios/talleres, clases prácticas, prácticas externas, tutorías, estudio y trabajo en grupo, estudio y trabajo autónomo.

Es interesante analizar cómo tanto la enseñanza presencial como la enseñanza virtual deben tener en cuenta este cambio metodológico, pero también es interesante comprobar cómo algunas herramientas digitales como las plataformas digitales, se preparan como recurso educativo tanto para la enseñanza presencial como para la enseñanza on-line de este nuevo planteamiento metodológico del Plan Bolonia.

\section{Adaptación de una plataforma al Plan Bolonia}

Si debemos llevar a cabo un cambio metodológico en la Enseñanza Superior (y en el resto de etapas, por supuesto), debemos adaptar o modificar los recursos que actualmente utilizamos.

Uno de estos recursos que cada vez se utiliza más en Educación Superior es una plataforma de e-learning o espacios personales de aprendizaje, tanto para la enseñanza a distancia como apoyo a la enseñanza presencial (intercambio de materiales, comunicación con alumnos...). Veamos a continuación qué debería tener una plataforma e-learning preparada para las nuevas metodologías de enseñanza y aprendizaje: 


\section{Aspectos funcionales}

- Creación de una comunidad educativa virtual: debemos tener presente la necesidad de hacer fácil el uso de la plataforma, que la comunidad educativa sienta que pertenece a una comunidad virtual al igual que pertenece a la institución en la que se ha matriculado. Ya se acceda directamente poniendo la clave del usuario en la web inicial o haya un enlace a la comunidad educativa y el usuario tenga que logarse en ella, solo se logará una vez en todo el proceso de acceso.

- Facilidad de acceso y navegación: una vez que el usuario se ha logado, debería ser fácil utilizar toda la plataforma, que el usuario no tenga problemas para localizar lo que busca, que sepa en todo momento dónde se encuentra, que tenga una opción de localización rápida (mapa)...

- Manual de uso y requisitos: al igual que proporcionamos al alumno una guía de estudios para el proceso de enseñanza y aprendizaje también debemos guiarle en la herramienta que va a utilizar como comunidad virtual. Pero además, no solo debe existir una guía para el alumno, sino una guía de uso para cada perfil de acceso, pues habrá alumnos, profesores, coordinadores, administradores..., que tendrán diversas opciones en función de su perfil. No solo debe existir un manual de uso, sino que además debe ser funcional.

Aspectos técnicos y estéticos

- Creación de contenidos: debe existir una herramienta de creación de contenidos en el perfil del docente, para que pueda crear contenidos educativos de diversas tipologías: subir documentos de distintos formatos y versiones, crear contenido SCORM directamente desde la plataforma, enlazar a un contenido externo, subir vídeos, audio...

- Creación de actividades: la plataforma debe admitir actividades de diversos formatos (hotpotatoes que es html, jclic que es zip, ejecutables, enlazar html...) y dar la posibilidad de crear wikis, formularios...

- Creación de biblioteca del conocimiento: en la plataforma es recomendable tener una zona personal de documentación útil para el usuario, una zona que organiza el propio usuario y que permite crear carpetas, ordenar, buscar... y en la 
que puedes subir documentos, traer documentos de las distintas asignaturas..., es como un disco duro del usuario.

- Catalogación de documentos: para poder realizar búsquedas fáciles y rápidas de la información, es necesario que los distintos elementos que se suban a la plataforma vayan siempre catalogados: título, materia, asignatura, curso, titulación, palabras clave, temática, fecha, autor...

- Creación de espacios colaborativos: con el Espacio Europeo de Educación Superior se hace especial hincapié en el trabajo colaborativo por parte del profesorado y del alumnado, por lo que deben existir herramientas que propicien este tipo de trabajo. Herramientas como el chat, el foro, el correo..., permiten el trabajo colaborativo, pero es recomendable que los usuarios de una plataforma asocien el trabajo colaborativo a una herramienta en concreto que puede ser una mezcla de funcionalidades de las otras, un aula virtual colaborativa.

- Herramientas de comunicación: es importante que existan múltiples herramientas de comunicación de fácil utilización: correo, foro, chat, blog, sistema de avisos a toda la comunidad educativa, sistema de avisos a los alumnos...

- Agenda: en esta nueva metodología en la que tanto profesorado como alumnado tienen una distribución heterogénea de horarios divididos en clases teóricas, clases prácticas, seminarios y tutorías esencialmente, se hace necesaria una agenda que permita poner citas globales a varios miembros de la comunidad educativa, poner citas personales cada uno en su perfil, ... De este modo, además, facilitamos que los profesores puedan saber la carga de trabajo que el alumno tiene para que sea más equilibrada entre asignaturas, y el alumnado puede organizar su tiempo en base a los trabajos que tiene programados en todo el curso.

- Tablón de anuncios: es recomendable que exista un tablón de anuncios general de la comunidad educativa y un tablón de anuncios por asignatura, de tal forma que puedan publicarse noticias, eventos, anuncios...

Aspectos pedagógicos

Muchos de los aspectos pedagógicos a tener en cuenta se han nombrado ya al mencionar los aspectos técnicos y estéticos, pero hablaremos de algún otro recurso: 
- Diseño de diversas modalidades educativas: en el actual proceso de enseñanza y aprendizaje deberían darse diversos tipos de modalidades educativas, ya sea una formación presencial, semipresencial o a distancia. Si utilizamos en cualquiera de dichas modalidades de formación una plataforma digital, deberíamos adaptar esta para que nos permitan introducir diversas modalidades educativas, al menos las que se tienen en cuenta desde el Plan de Bolonia:

o Clases teóricas: sesiones expositivas, explicativas y/o demostrativas de contenidos. Esta opción trasladada a una plataforma digital, requiere de esta una herramienta para poder compartir documentos con los alumnos de diversos formatos (pdf, powerpoint, html, scorm) y de diversas versiones.

- Clases prácticas: cualquier tipo de práctica de aula. En una clase práctica presencial, el alumnado pone en práctica los conocimientos adquiridos a través de estudios de casos, análisis diagnósticos, problemas de laboratorio... En una plataforma digital esta modalidad de enseñanza debe permitirnos crear una actividad que el alumno pueda realizar directamente, o permitirnos darle unas orientaciones para que haga la práctica y luego responda en la plataforma a unas cuestiones, debe permitirnos poner vídeos de la práctica $o$ enlaces donde poder visualizar la práctica para que luego la lleven a cabo los alumnos o la analicen...

o Seminarios/talleres: sesiones monográficas supervisadas con participación compartida (profesores, alumnos, expertos...). En educación presencial los seminarios suelen consistir en actividades de intercambio de conocimientos en pequeños grupos, por lo que en la plataforma se requiere de herramientas que permitan agrupar a los alumnos de distinta forma según el seminario, y que puedan interactuar para trabajar alumnos, profesores, expertos... Es muy útil en este sentido que la plataforma cuente con la herramienta de chat y con la posibilidad de crear varias salas intercambiando ideas, documentos, enlaces... Esta modalidad educativa también es posible a través de la videoconferencia, por lo que es una herramienta a tener en cuenta en una plataforma educativa.

- Tutorías: relación personalizada de ayuda en la que un profesor-tutor atiende, facilita y orienta a uno o varios estudiantes en el proceso formativo. La plataforma digital debe permitir que profesorado y alumnado se comuniquen fácilmente, a través del correo, chat o incluso una herramienta específica de tutoría que permite solicitar tutoría con el profesor o el profesor 
con el alumno, que permite comunicación bidireccional, que guarda las conversaciones llevadas a cabo, que permite intercambiar escritorios, ver a la persona con la que te estás comunicando,...

o Prácticas externas: formación realizada en empresas y entidades externas a la universidad. En la educación superior una de las materias a cursar es la realización de prácticas externas a la institución. En este caso, debemos tener a nuestra disposición una herramienta que nos permita la gestión de estas prácticas, crear listados de alumnos, listados de centros, intercambiar documentos, comunicación con los centros... O también podemos utilizar las herramientas mencionadas anteriormente y agrupadas en una zona de "Prácticas externas" como: subir documentos de diversos tipos y versiones, posibilidad de compartir documentos con quien el usuario quiera, posibilidad de escribir correos, posibilidad de crear una sala de chat para dialogar con el centro de prácticas (atención, es un usuario externo a la plataforma pero le invito),...

- Estudio y trabajo en grupo: preparación de seminarios, lecturas, investigaciones, trabajos, memorias, obtención y análisis de datos, etc. para exponer o entregar en clase mediante el trabajo de los alumnos en grupo. Es imprescindible en este sentido que la plataforma educativa que se utilice tenga herramientas colaborativas que permitan a los alumnos trabajar en equipo: posibilidad de crear grupos de discusión, posibilidad de crear salas de chat, posibilidad de compartir trabajos, posibilidad de trabajar varios en un mismo documento a la vez,...

- Estudio y trabajo individual: el estudio personal (preparar exámenes, trabajo en biblioteca, lecturas complementarias, hacer problemas y ejercicios, etc.) es fundamental para el aprendizaje autónomo. Esta metodología educativa en una plataforma virtual se ve representada sobre todo en la biblioteca del conocimiento del alumno, donde puede encontrar toda la información que necesita para su trabajo diario en las asignaturas. Además, al estar catalogada toda la información, el alumno puede realizar búsquedas sencillas que le faciliten la localización de la información.

- Contenidos educativos: se deben poder compartir los contenidos educativos y cualquier elemento subido a la plataforma, con el resto de usuarios, para lo cual cada usuario tendrá una opción de compartir recurso y entrará a formar parte de la biblioteca del conocimiento, delimitando si el usuario quiere compartirlo con 
toda la comunidad educativa, con sus compañeros, con los profesores, o con un grupo en concreto.

- Actividades educativas: para evaluar las actividades el profesor puede elegir el sistema de calificación y siempre podrá ponerle comentarios a los alumnos y decidir qué actividades son evaluables y cuáles no.

- Herramientas de evaluación: debemos disponer en la plataforma de diversas herramientas de evaluación según su finalidad:

o Del aprendizaje: pretendemos evaluar el aprendizaje de los alumnos, para lo cual podemos utilizar diversas actividades, exámenes, formularios, tiempo conectado, participación en foros... Sería bueno que los profesores pudieran elegir qué es evaluable y en qué grado influye en la nota final. Además, siempre que algo es evaluable debe permitir, poner nota numérica (elegida la escala por el profesor), un comentario y añadir un archivo. Además de la evaluación de las actividades que se convertirán en una nota final, debe existir otra herramienta de evaluación paralela en la que cada profesor marca si ha sido adquirida cada una de las competencias que debía de adquirir el alumno en cada asignatura y algún comentario que el alumno puede o no ver, en función de lo que decida el profesor. Esta herramienta tendrá asociadas las competencias de las asignaturas a las competencias de las materias, por lo que cuando finalice la titulación, al alumno se le proporcionará un informe de evaluación de las competencias en las que ha estado involucrado en función de su titulación. Además, para evaluar las competencias generales se procederá de la misma forma, pero existirá una evaluación final de todo el profesorado y se verá reflejado en ese informe final de competencias.

- De la enseñanza: debe existir también una herramienta para evaluar la enseñanza, esencialmente al profesor, a la asignatura y a la institución/ plataforma. Esta herramienta será tipo test con alguna pregunta abierta.

A grandes rasgos, estas son algunas de las características deseables en un espacio personal de aprendizaje virtual que esté preparado para la educación superior en el Espacio Europeo.

Son muchas las plataformas educativas que pretenden ofrecen estos espacios personales de aprendizaje, pero no todas lo consiguen, y si miramos la calidad de las mismas, se nos reduce aún más el número de ellas. 
Si damos importancia a la evaluación de la calidad de otros recursos, también debemos darle importancia a la evaluación de la calidad de los recursos digitales de enseñanza y aprendizaje, pues, hoy son un recurso vital y seguro en la educación.

\section{NOTAS}

$1 \quad$ Gisbert y otros (1998). En: CEBRIÁN y otros (1998).

2 Comisión de las Comunidades Europeas. Comunicación de la Comisión al Consejo y al Parlamento Europeo. Plan de acción eLearning. Concebir la educación del futuro. Bruselas, 28.3.2001. COM (2001) 172 final.

3 Blázquez (1998). En: Díaz-Sanchez, F. A. (1999).

4 Extraído de "Los instrumentos de la Unión Europea relativos a la relación de las TIC a la educación y a la formación”. [En Red]. http://www.ilo.org/public/spanish/ employment/skills/recomm/topic i/t8 eu.htm

5 Comisión de las Comunidades Europeas. Proyecto de programa de trabajo detallado para el seguimiento del informe sobre los objetivos concretos de los sistemas de educación y formación (Comunicación de la Comisión). Bruselas, 07.09.2001. COM (2001) 501 final.

\section{REFERENCIASBIBLIOGRÁFICAS}

Benito, A.; Cruz, A. (2005). Nuevas claves para la docencia universitaria en el espacio europeo de educación superior. Madrid: Narcea.

Colás Bravo, C.; De Pablos, J. (Coord.). (2005). La Universidad en la Unión Europea: el Espacio Europeo de Educación Superior y su impacto en la docencia. Málaga: Aljibe.

Díaz Sánchez, F. A. (1999). Usos educativos de las nuevas tecnologías. Granada: GEU.

Cebrián, M. (Coord.). (1998). Recursos Tecnológicos para los Procesos de Enseñanza y Aprendizaje. Málaga: ICE/Universidad de Málaga.

Levy-Leboyer, C. (1997). Gestión de las competencias. Barcelona: Gestión 2000.

De Miguel Díaz, M. (Dir.). (2005). Modalidades de enseñanza centradas en el desarrollo de competencias. Orientaciones para promover el cambio metodológico en el Espacio Europeo de Educación Superior. [en línea] Disponible en: http://www. ub.edu/oce/documents/pdfes/mec/ mec 2005 comp.pdf (consulta 2010, 1 de junio).

Massot, P.; Feisthammel, D. (2003). Seguimiento de la competencia y del proceso de formación. Madrid: Ediciones Aenor.

McClelland (1973). Testing for competence rather Than For Intelligences. [en línea] Disponible en: http://www.lichaoping. com/wp-content/ap7301001.pdf (consulta 2010, 1 de junio).

Roig, R. (2002). Las nuevas tecnologías aplicadas a la educación. Alicante: Marfil.

Tobón, S; García-Fraile, J. A.; otros. (2006). Competencias, calidad y educación superior. Bogotá: Magisterio.

Yáñiz, C.; Villardón, L. (2006). Planificar desde competencias para promover el aprendizaje. Bilbao: Universidad de Deusto.

Zabalza, M. (2003). Competencias docentes del profesorado universitario. Madrid: Narcea. 


\section{PERFIL ACADÉMICO Y PROFESIONAL DE LAS AUTORAS}

Inmaculada Tello Díaz-Maroto. Psicopedagoga. Doctora en Educación. Profesora Titular de la Escuela Universitaria Cardenal Cisneros.

E-mail: inmacualda.tello@cardenalcisneros.es

Lourdes de Miguel Barcala. Pedagoga. Profesora Titular de la Escuela Universitaria Cardenal Cisneros.

E-mail: lourdes.demiguel@cardenalcisneros.es

María Dolores López Carrillo. Bióloga. Doctora en Geología. Profesora Titular de la Escuela Universitaria Cardenal Cisneros.

E-mail: lola.lopez@cardenalcisneros.es

DIRECCIÓN DE LAS AUTORAS

Escuela Universitaria Cardenal Cisneros

Avda/Jesuitas, 34,

28806, Alcalá de Henares, España

Fecha de recepción del artículo: 09/9/11

Fecha de aceptación del artículo: 03/11/11

\section{Como citar este artículo:}

Tello Díaz-Maroto, I.; de Miguel Barcala, L.; López Carrillo, M. D. (2012). Entornos personales de aprendizaje en el Espacio Europeo de Educación Superior. RIED. Revista Iberoamericana de Educación a Distancia, volumen 15, $\mathrm{n}^{0}$ 2, pp. $123-142$. 